\title{
An EDF Interrupt Handling Scheme for Real-Time Kernel: Design and Task Simulation
}

\author{
Peng Liu ${ }^{1}$, Ming $\mathrm{Cai}^{1}$, Tingting $\mathrm{Fu}^{2}$, and Jinxiang Dong ${ }^{1}$ \\ ${ }^{1}$ Institute of Artificial Intelligence, Zhejiang University, Hangzhou, PRC, 310027 \\ \{perryliu,cm,djx\}@zju.edu.cn \\ ${ }^{2}$ Institute of Graphics and Image, Hangzhou Dianzi University, Hangzhou, PRC, 310018
}

\begin{abstract}
Traditional model of interrupt managemnent has been used for several decades. But it is often incapacity to incorporate reliability and temporal predictability demanded on real-time systems. Many solutions have been proposed to improve efficiency of interrupt handling such as In-line Interrupt Handling and Predictable Interrupt Management In this paper we propose a model that schedules interrupts in terms of their deadlines. Hard priorities of IRQs are still left to hardware. We only manager interrupts that can enter the kernel space so that hard real-time can be assured. Each interrupt will be scheduled only before its first execution according to their arrival time and deadlines so that the scheme is very simple and easy to be implemented. The scheme tries to make as many as possible ISRs finish their work within the time limit. Finally we do some experiments, which prove there is a big decrease of nested overtime interrupts, by means of task simulation on VxWorks.
\end{abstract}

Keywords: Real-time system, Interrupt scheduling, Task simulation, Similar Earliest-Deadline-First.

\section{Introduction}

Most embedded systems include a lot of external devices. The interrupt mechanism is a very important interface between the kernel and peripherals, which communicate the system with its external environment. Too many interrupt sources will cause the number of expired ISRs getting too high. It will also cause potential system instability or inconsistency. For example, as seen in fig. 1(left chart), $\mathrm{I}_{1}, \mathrm{I}_{2}, \mathrm{I}_{3}$ are three interrupt routines which priorities meet $\mathrm{PI}_{1}<\mathrm{PI}_{2}<\mathrm{PI}_{3}$. Their trigger time and endurance time can be seen in table 1. According to traditional scheme of interrupt management $I_{1}$ is preempted by $I_{2}$ even if it is about to finish its work. And $I_{2}$ is preempted by $I_{3}$ twice in succession. So $I_{1}$ completes its work at moment 15 . Because its endurance time is 3 its work has been made no sense. $\mathrm{I}_{2}$ has been overtime as well.

A number of research works propose alternatives to avoid the difficulties of the traditional interrupt model for real-time applications. Some have adopted radical solutions where most external interrupts are disabled and treat all peripherals by polling [1]. Other strategies have been proposed to obtain some degree of integration among the different types of asynchronous activities [2]. In [3], interrupts are treated as threads. In [4], a schedulability analysis integrating static scheduling techniques and 
response time computation is proposed. In [5] an integrated scheme of tasks and interrupts to provide predictable execution times to real-time systems is presented. In this paper, we propose a novel strategy that manages interrupts using EDF scheduling. It is supposed to avoid many drawbacks of above schemes.

Table 1. Four ISRs (Tasks) invoked in the system

\begin{tabular}{ccccc}
\hline IRQ Number & Arrival T & Service T & Max. alive T & Priority \\
\hline $\mathrm{I}_{1}\left(\mathrm{~K}_{1}\right)$ & 1 & 3 & 3 & $\mathrm{PI}_{1}\left(\mathrm{PK}_{1}\right)$ \\
$\mathrm{I}_{2}\left(\mathrm{~K}_{2}\right)$ & 3 & 7 & 10 & $\mathrm{PI}_{2}\left(\mathrm{PK}_{2}\right)$ \\
$\mathrm{I}_{3}\left(\mathrm{~K}_{3}\right)$ & 8 & 2 & 4 & $\mathrm{PI}_{3}\left(\mathrm{PK}_{3}\right)$ \\
$\mathrm{I}_{3}\left(\mathrm{~K}_{3}\right)$ & 11 & 2 & 4 & $\mathrm{PI}_{3}\left(\mathrm{PK}_{3}\right)$ \\
\hline
\end{tabular}
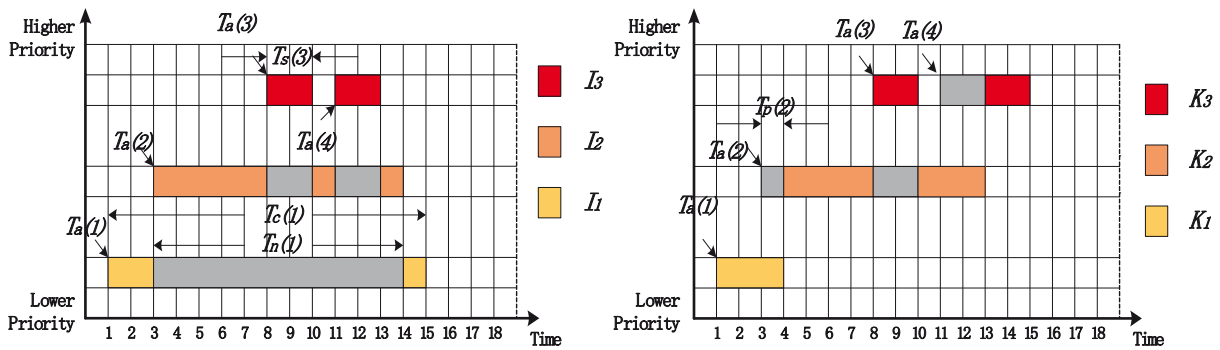

Fig. 1. Results of two interrupt management schemes

\section{Model and Mechanism}

Earliest-Deadline-First algorithm is a dynamic scheduling scheme. Priorities of tasks are dynamic according to their start-time and deadline. Those whose deadline is nearest to the current time have higher priorities. Priorities should be calculated again after the end of a task. The last step of scheduling is to choose a ready task that has highest priority.

Our scheme is a Similar EDF algorithm which sorts interrupt service routines according to three factors: hard priority, arrival time and deadline. Only when higher ISRs have spare time to allow lower ISRs to finish their work the scheduling will happen. Otherwise higher ISRs will preempt lower ones even though they may be overtime. Furthermore every ISR will be scheduled at most once for the sake of simplicity. Just like a stack, once an ISR is scheduled to run as if it is put into "stack". Others may preempt it as put into "stack" above it. Only when it is the top item of the "stack" can it be performed again. In our scheme following properties are considered: Processor availability A(i); Maximum alive time (deadline) $\mathrm{T}_{\mathrm{b}}(\mathrm{i})$; Arrival time $\mathrm{T}_{\mathrm{a}}(\mathrm{i})$; Service time $\mathrm{T}_{\mathrm{s}}(\mathrm{i})$; Execution time $\mathrm{T}_{\mathrm{e}}(\mathrm{i})$; Nested time $\mathrm{T}_{\mathrm{n}}(\mathrm{i})$; Hard Priority PI(i)

Maximum alive time means how long the ISR could stay in the system to finish his work before the deadline. Arrival time denotes when the interrupt is triggered. Service time means how long it would take for an ISR to complete a mission. Execution time indicates how long the ISR has taken to do its work. Nested time represent the period that the ISR is preempted by others. So we have: 


$$
\begin{gathered}
\mathrm{U}(\mathrm{t})=\operatorname{Min}\left(\left(\mathrm{U}(\mathrm{t}-1)-\mathrm{T}_{\mathrm{s}}(\mathrm{i})\right),\left(\mathrm{T}_{\mathrm{b}}(\mathrm{i})-\mathrm{T}_{\mathrm{s}}(\mathrm{i})\right)\right) \\
\mathrm{T}_{\mathrm{e}}(\mathrm{i})<=\mathrm{T}_{\mathrm{s}}(\mathrm{i})
\end{gathered}
$$

An interrupt $\mathrm{j}$ can be scheduled only and only if:

$$
\mathrm{T}_{\mathrm{b}}(\mathrm{j})-\mathrm{T}_{\mathrm{s}}(\mathrm{j})>=\mathrm{T}_{\mathrm{s}}(\mathrm{i})-\mathrm{T}_{\mathrm{e}}(\mathrm{i})
$$

We encapsulate the scheduling codes and user ISR into a code segment called realIntISR and use it to replace user ISR. It includes entering scheduling logic and exiting scheduling and is transparent to users.

\section{Task Simulation and Performance}

Here we have three tasks to simulate three interrupts $I_{1}, I_{2}$ and $I_{3}$ respectively on VxWorks. Their parameters are set as shown in table 1. We first create three tasks synchronously. Then put them into sleep until their pre-set trigger time is matched. Tasks just print message "intX is executing" onto the screen every second. We augment time scale to second to see the result clearly. After testing of the task simulation, the original system brings a result like what is shown in fig. 1(left chart). As expected, $\mathrm{I}_{1}$ (simulated by $\mathrm{K}_{1}$ ) is preempted by $\mathrm{I}_{2}$, then by $\mathrm{I}_{3}$ and $\mathrm{I}_{4}$. In improved system, EOI will be sent after scheduling immediately for not to prevent the same level interrupt from entering. As shown in fig. 1(right chart), the total time consumed by three simulated task is not changed, but all three interrupts finished their work in time while two of them failed in original system.

The performance of the algorithm is tested also using task simulation. In each ISR, execution timer is recorded. If it is overtime a global count will be increased. We set five tasks which can be seen in table 2 .

Table 2. Parameters of five tasks

\begin{tabular}{llllll}
\hline IRQ Number & 1 & 2 & 3 & 4 & 5 \\
\hline Service time & $990 \mathrm{~ms}$ & $150 \mathrm{~ms}$ & $1.9 \mathrm{~ms}$ & $1.8 \mathrm{~ms}$ & $1 \mathrm{~ms}$ \\
Lifecycle(Deadline) & $4800 \mathrm{~ms}$ & $600 \mathrm{~ms}$ & $6 \mathrm{~ms}$ & $6 \mathrm{~ms}$ & $4.2 \mathrm{~ms}$ \\
Interrupt Frequency & $12 / 60 \mathrm{~s}$ & $60 / 60 \mathrm{~s}$ & $3000 / 60 \mathrm{~s}$ & $3000 / 60 \mathrm{~s}$ & $4200 / 60 \mathrm{~s}$ \\
\hline
\end{tabular}

We keep those tasks running for a fixed period on the original and improved systems respectively. The result can be seen in fig. 2 . The left chart shows new algorithm can do more interrupts than traditional algorithm in an equal period. The right chart shows new algorithm has less overtime interrupts than traditional algorithm. In conclusion, our scheme can improve the performance about $30 \%$ in situation that there are a good many interrupts and most of them have limited execution time while some of them could wait for execution for some time. Due to additional code introduced by the scheme, the performance of the system maybe decreases when a lot of interrupts have very short service time and restricted maximum alive time. 

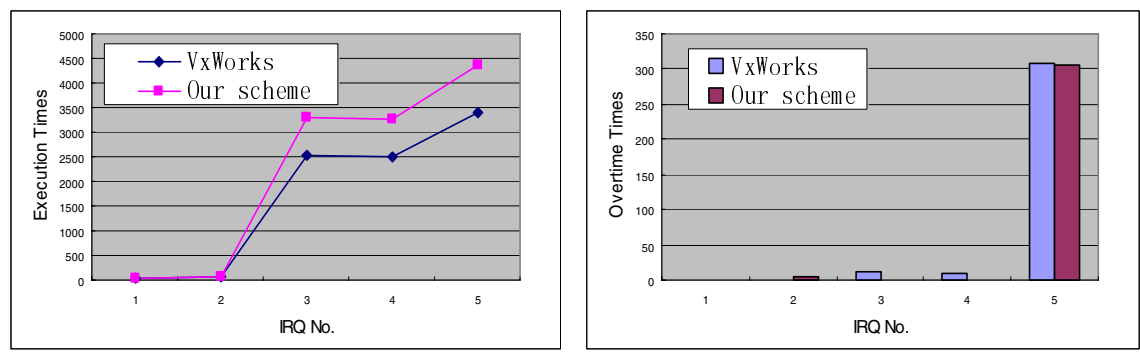

Fig. 2. Performance contrast

\section{Conclusion}

Most embedded systems have many interrupt sources and these interrupts will occur asynchronously. When there are too many nested interrupts those in low level are likely to run out of time, which leads to failure of their work. In this paper we presented a Similar Earliest-Deadline-First handling scheme to provide schedulability to interrupt management of real-time systems. Its algorithm and architecture were discussed. A simulation using tasks was presented. Result of performance test, which was carried out based on that simulation, was given. It was proved that using Similar EDF scheduling could greatly reduce interrupt failure caused by nested interrupts and enhance robustness of the real-time system.

\section{References}

1. Hermann, K., et-al.: Distributed Fault-Tolerant Real-Time Systems: the MARS Approach. IEEE Micro, Vol. 9, Issue 1. (1989) 25-40

2. Tullio, F., et-al.: Non-Preemptive Interrupt Scheduling for Safe Reuse of Legacy Drivers in Real-time systems. In: Proceedings of the 17th EuroMicro Conference on Real-Time Systems. (2005) 98-105

3. Steve, K., Joe, E.: Interrupts as Threads. ACM SIGOPS Operating Systems Review, Vol. 29, Issue 2, (1995) 21-26

4. K, Sanstrom., C, Erikssn., G, Fohler.: Handling Interrupts with Static Scheduling in an Automotive Vehicle Control Systems. In: Proceedings of 5 International Conference on Real-Time computing Systems and Applications. (1998) 158-165

5. Luis E, L-d-F., Pedro, M-A., Dionisio de, N.: Predictable Interrupt Management for Real Time Kernels over Conventional PC Hardware. In: Proceedings of IEEE Real-Time and Applications Symposium. (2006) 14-23

6. Aamer, J., Bruce, J.: In-Line Interrupt Handling and Lock-Up Free Translation Lookaside Buffers (TLBs). IEEE Transaction on Computers, Vol. 55. (2006) 559-574 